\title{
An Implementation of a Point of Care Information System for Nurses
}

\author{
Terungwa Simon Yange \\ Department of Mathematics/Statistics/Computer \\ Science \\ University of Agriculture, Makurdi
}

\author{
Onyekware U. Oluoha \\ Department of Computer Science \\ University of Nigeria \\ Nsukka
}

\begin{abstract}
Point-of-care data capture is an important part of a clinical information system. Unfortunately, health care providers find it time consuming and repetitive to perform data entry via computers. In this paper, we designed a user-friendly interface for the entry of patient information which gives the health care provider an easy to use interface for patient data entry. This system uses portable terminals and supports the entire loop of daily nursing work. This system was developed with the aim to make nursing work more efficient, by reducing the time needed for indirect work (patent information documentation, conferring with other healthcare providers, etc.), while increasing the time expended in direct patient care. The proposed system has three main functional parts: 1) Data Browsing which provides patient information such as a brief history, assessments and vital-sign charts; 2) Schedule Planning helps nurses organize doctors' instructions and make To-do lists for the day; and 3) Care Management which reminds nurses when they should execute the doctors' orders and provides tools for data entry. These three functions cover the entire loop of daily nursing work. Results from a preliminary hospital evaluation showed that use of the system can reduce the time needed for nursing documentation by $60 \%$ (a $10 \%$ saving of a nurse's total working time), and that more than $80 \%$ of the nurses who used the system felt it would make nursing work more efficient.
\end{abstract}

\section{Keywords}

Point of care, care, time-varying, nursing, nursing process, nursing care plan

\section{INTRODUCTION}

Research has shown that the deployment of Information technology in healthcare provision can greatly increase the efficiency, decision-making and effectiveness of healthcare providers [1]. However, healthcare providers/professionals seem reluctant to perform clinical data entry for numerous reasons [2], including the believe that using computers during point of care can be repetitive than traditional paper-based forms, that Information Communication Technology (ICT) are equally difficult to use and require more time and expertise or they may not have a computer readily available at the point of care [3]. Most structured data entry systems in particular are objected to because they interfere with the clinician's thinking process. These systems may require the user to map their own concepts into the computer and to spend time searching for the "right" computer code or phrasing, or force users to navigate a singular hierarchy of structured data. Providing users with multiple data entry options may help overcome this obstacle.

According to [4], care is defined as the services rendered to the patient by healthcare professionals; and point of care is the location at which patient services are delivered by these professionals. Point-of-care therefore, refers to the products and services delivered to the patient at bedside. These include laboratory and other services provided to patients at the bedside. During this process, data is generated and documented.

Documentation performed at the clinical point of care can be conducted using either paper, electronic formats or both. This process aims to capture medical information pertaining to patient's healthcare needs [5]. The patient's health record is a legal document that contains details regarding patient's care and progress. The types of information captured during the clinical point of care documentation include the actions taken by clinical staff including physicians and nurses, and the patient's healthcare needs, goals, diagnosis and the type of care they have received from the healthcare providers [6][7].

Such documentations provide evidence regarding safe, effective and ethical care and insinuates accountability for healthcare institutions and professionals [8]. Furthermore, accurate documents provide a rigorous foundation for conducting appropriate quality of care analysis that can facilitate better health outcomes for patients. Thus, regardless of the format used to capture the clinical point of care information, these documents are imperative in providing safe healthcare. Also, it is important to note that electronic formats of clinical point of care documentation are not intended to replace existing clinical process but to enhance the current clinical point of care documentation process.

Activities at the bedside are divided into various situations with distinct characteristics. Some are scheduled routines (e.g., morning meetings, patient rounds, etc.), while others are unplanned (e.g., incidental face-to-face meetings, patient related events requiring immediate attention). In all these, huge data is captured from the patient and this data changes with time.

This research primarily focused on situations in which nurses located at the patient bedside are able to capture data which automatically update what is in the database at the server. The main reason why nurses are considered is because of the closeness to and easy access by the patient.

Nurses are the health professionals that are closest to, and spend most of their time with patient. The nursing profession is dynamic and so is the data captured in the course of their activities [1]. Therefore, the storage structure must take into cognizance this fact (time-varying) [9][10]. In this paper, we focused on the development of a system for managing nursing data; since it is highly dynamic and hence, very difficult to manage electronically.

\section{RELATED WORKS}

Numerous scholars surmised that information technology can greatly improve the provision of nursing care [11][12]. The use of computers by nurses in the course of the execution of 
their work at bedside seeks to increase their productivity by reducing paperwork, making information more accessible, facilitating communication among healthcare team members and improving the quality nursing documentation [13]. It is evident that nurses need not only understand nursing information systems, but also to use them effectively and apply the knowledge gained in rendering quality patient care. However, there appear to be a sense of despondency amongst professional nurses regarding the effective use of computerbased systems in general [4]. Although computer-based nursing information systems have a promising future for nursing profession, it still suffers rejection due to the fact that they lack features that capture the true picture of nursing.

According to [14], changes in the healthcare settings are regular occurrences; and the computerization of nursing systems is one of such changes taking place in the information revolution. Although, nurses have widely varying attitudes toward computers and changes in the workplaces, the transition of nursing team effectively from the manual system to the computerized systems has some impediments which include:

i. Inability to manage nursing process record efficiently;

ii. Irrelevance to patient care; and

iii. Inability to generate meaningful clinical outcomes.

The researcher further presented a theoretical framework based on how Lewin's Force Field Analysis Model could be applied in the practice settings to implement a nursing information system successfully.

[1] studied the clinical nurses' and midwives' perceptions and understandings of computerized information systems in everyday practice. The study focuses specifically on clinicians' experiences of using Computerized Patient Information Systems (CPIS) to manage clinical information. Clinicians' experiences were characterized by digital disappointment rather than electronic efficiencies. They reported generally that computerization had neither enhanced their clinical practice nor patient care, nor had it improved patient outcomes. The participants' experiences were predominantly negative and mostly critical of CPIS and their perceived inability to capture 'real nursing', difficulty in use, incompatibilities, non-responsiveness and irrelevance to patient care and meaningful clinical outcomes. Although the computerization of nursing activity will continue to impact upon clinical practice and cannot be wished away, but most systems developed today have little or no regard for clinician end-users. A crucial issue facing everyone in health informatics is how patient care systems can be developed in ways that involve clinicians meaningfully and which recognize and respond to the complexity and subtlety of the world of nursing and midwifery practice [15].

In a survey carried out by [16] on the challenges facing nursing practice in Nigeria, the dynamic nature of nursing profession and poor information systems were the major issues identified as hindering development of nursing service. Information available to both the patient and the nurse is very poor as a result there is difficulty in keeping and managing nursing history. Therefore, data available are not reliable and accurate leading to challenges in proper planning for patient care.

[17] After a survey of several nursing informatics frameworks reached at the following conclusions:

i. Most nursing informatics frameworks do not include a dimension or aspect of time and this caused some difficulties since nursing activities depend solely on time;

ii. Most nursing informatics frameworks lack environmental and task-oriented elements that are essential to understanding computer interaction; and

iii. Elements of nursing informatics frameworks are conceptualized differently across different platforms.

These leads to the development of systems that lack the following features:

i. Prompts or reminders within the period of care process (i.e., when to carryout assessments, diagnosis, plan for care, implement the care planned and evaluation)

ii. Ability to collect real time nursing information

iii. Standardized and streamlined diagnoses and interventions

iv. Keeping of past and future records

The views of [17] were supported by [4] while evaluating the effective use of computer-based nursing information systems in patient care by professional nurses.

\section{METHODOLOGY}

As stated at the beginning, the aim of this paper is to design a data capturing system for nurses at point of care. To achieve this, the system was designed using unified modelling language (UML) tools. The design includes use case diagram (Fig. 1), the class diagram (Fig. 2) and the activity diagram (Fig. 3). Nurses employ the nursing process in the course of delivery of their services at the point of care, the design hinges around the five phases of the framework (assessment, nursing diagnosis, planning, implementation and evaluation). The assessment phase in this system is modelled using the Gordon's eleven functional health patterns; at the planning phase, we prioritize the patient's need using the Maslow's hierarchy of needs. The linkage between the NANDA International (NANDA-I) diagnoses, the nursing outcome classification (NOC) outcomes and the nursing interventions classifications (NIC) interventions was used. This linkage is popularly known as the NNN. This language establishes the relationship between the standard diagnoses used in the diagnosis phase, the standard outcomes used to define both the expected outcomes and the actual outcomes, and the standard interventions used in the intervention phase.

The designed system was implemented using the Java Programming language (Java platform Enterprise Edition) to develop the business logic and the presentation, and the backend was implemented using the structured query language (PostgreSQL to be precise). This system is called 'eNCMS'- electronic Nursing Care Management System.

\section{RESULTS AND DISCUSSION}

The $e N C M S$ is an enterprise/web-based application developed to enable nurses to capture patient data collected during the implementation of nursing process at point of care. This system provides an efficient approach that improves the quality of nursing care and proper documentation which enhances nursing productivity; and as well provide a database 
for nursing research and quality assurance in nursing. This system is very easy to use, allowed nurses' freedom of expression patient chart, and maintained links to NANDA-I, NOC, and NIC codes. To achieve these, we specifically paid attention to

i. Design the interface elements from the clinician's perspective.

ii. Keep inter-window navigation and scrolling to a minimum.

iii. Reduce keyboard entries and mouse clicks to a minimum.

iv. allow complete entry using either the mouse, or the keyboard, exclusively, or in combination.
Listed below are some screenshots from the system. Fig. 4 shows the deployment view for this system; Fig 4-10 shows some of the screenshots for capturing assessment data. Figure $4 \mathrm{f}$ and $4 \mathrm{~g}$ shows the nursing care plan for a patient.

Fig 5-10, shows some of the screenshots from the system. The vital signs (temperature, pulse and respiration-TPR) as recorded in the database for a patient recorded within the period of five (5) hours at different intervals are shown in fig $8-10$. This shows the variation in the states of data collected by nurses at the point of care. These vital signs were recorded eleven (11) times within this period (5hours).

From our findings, most point of care data entry for nurses were not adopted because of their inability to properly handle nursing activities at patient bedside, most especially the dynamic nature of the nursing process [4][17][18].

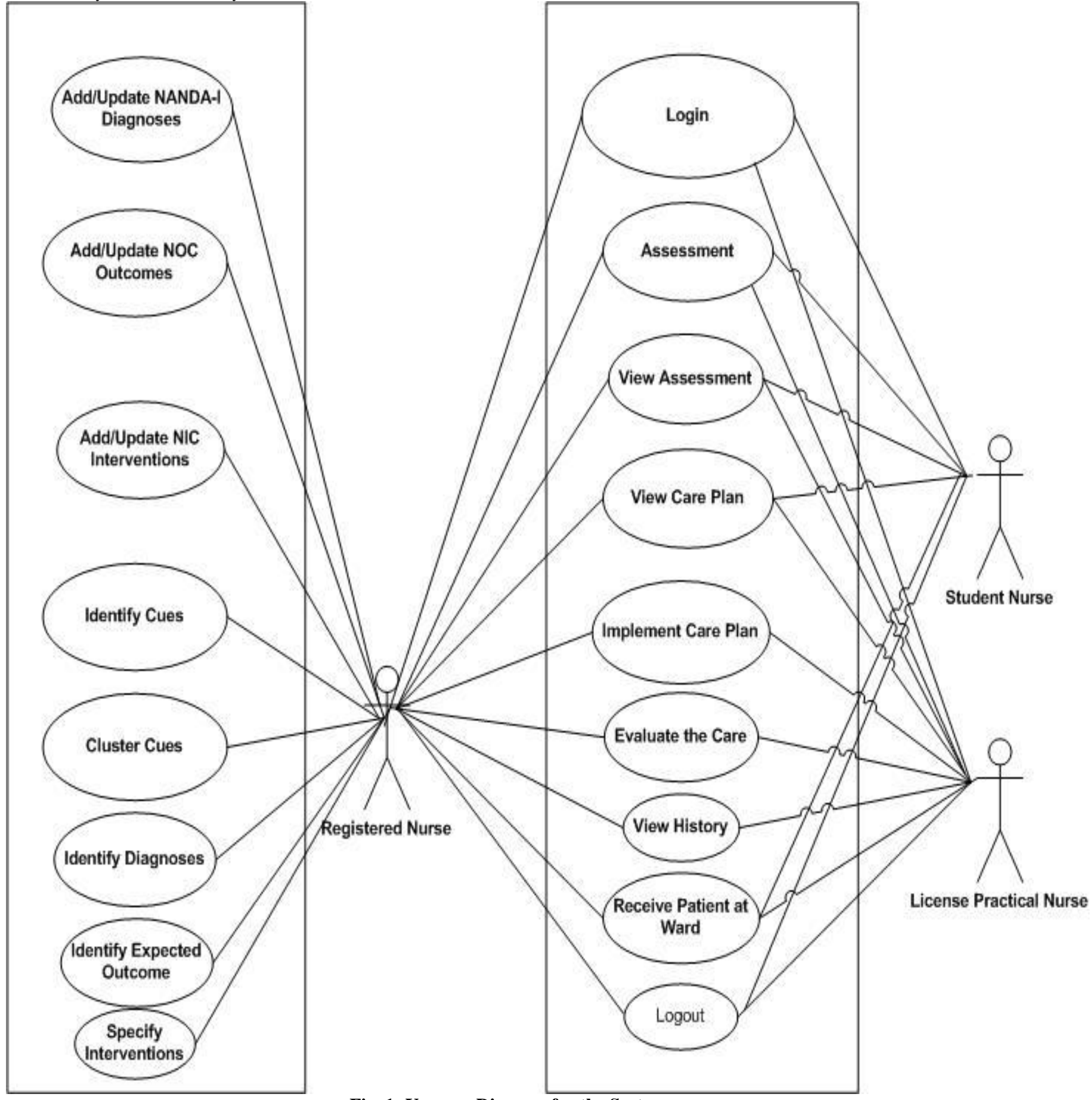

Fig. 1: Use case Diagram for the System 


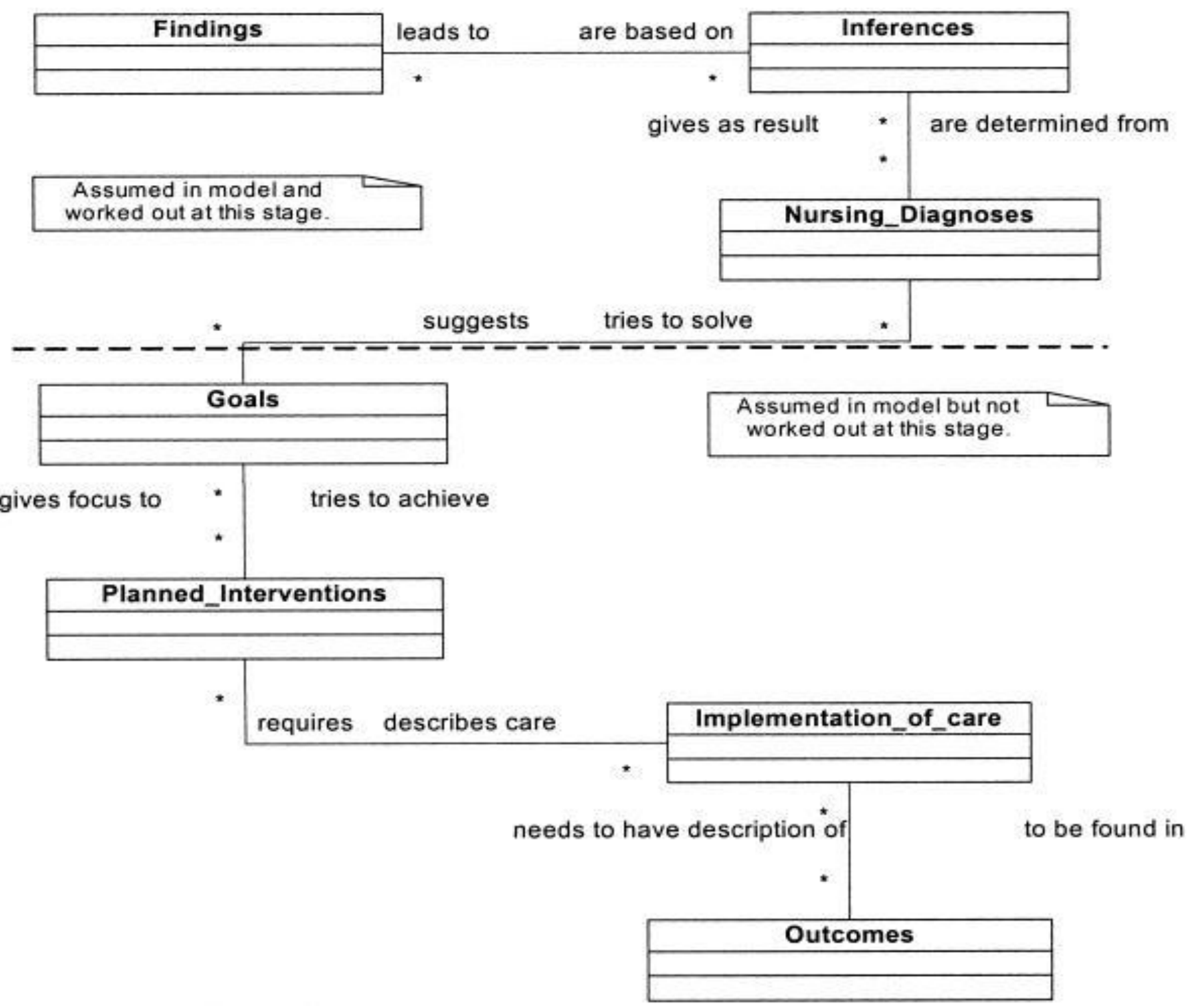

Fig. 2: Class Diagram

This was as a result of the fact that these systems where built on data models which have little or no support for the dynamic changes of data [16][17]. By this, handling timebounded data becomes very difficult with these systems and therefore, nurses document their data using paper forms. The paper adopted the temporal data model designed by [19]. This aid it in checkmating most of the issues pointed out as the deterring factors by the other researchers above.

For a system to fully work in a nursing setting, it should be able to handle temporal behaviour of data i.e., it should be able to handle past data (history), present and/or future data as the case may be. With that, it will be very easy to keep nursing history, data collected during the implementation of nursing process will be properly managed, and it will be very easy to implement the long-awaited dream of nurses-the electronic health record. In order to build such a system, one need to first and foremost extend an existing data model so that it will support temporal data since existing model do not or have little support for time-varying data. When this is done, the extended data model can then be implemented so as to come up with the desired system.

This research implements an object-relational data model called the nursing care temporal object-relational data model which is an extension of object relational data model [19].
This model allows us to define the data types for our data using abstract data type defined in the implementation of the model. This makes it easier to manipulate the time-varying nature of the data through timestamping. The model is bitemporal in nature because it accepts data timestamped with both transaction time and valid time. By this, past, present and/or future data could be fully implemented. The implementation of this model gave birth to the eNCMS.

The $e N C M S$ is a web-based distributed system designed to address the problem nurses encounters during the documentation of patient records. The system supports adequate client demographic data, enhances workflow and allows authorized users to manage (i.e., retrieve, update, delete, etc.) client information according to their level of authentication.

The incorporation of the NNN linkage in this system is a major advancement in facilitating the use of these standardized nursing languages in practice, education and research. This has developed a template to build a knowledge base that supports professional practice and improves the quality of care provided by nurses in a variety of settings across the health care continuum. This has addressed the need for representation and classification of the knowledge base of nursing. Most important to this is the knowledge of nursing 
diagnoses, patient outcomes, and nursing interventions. This has increased the practicality and efficiency of managing nursing data.

The system was tested using five (5) patient data collected from Obafemi Awolowo University Teaching Hospital Complex (OAUTHC), Ile-Ife, Nigeria. The test data was evaluated using both white box testing and black box testing model called modified Musa basic execution time model. In the white box testing, a total of six (6) errors were detected and using the model, another error was discovered, and because this error was not caught and corrected during the actual testing, we have a reliability of $0.8571(85.71 \%)$. For the black box testing, a total of six (6) errors were also detected and using the model, two (2) more errors were uncovered; and because these two (2) errors were not corrected during the actual testing, we have a reliability of $0.75(75 \%)$. With the result of these two (2) testing methods, it shows that the software is very reliable.

\section{CONCLUSION}

In conclusion, an efficient nursing care data entry system which provides the mechanism to capture the dynamic data nurses capture at point of care was developed. The entity relationship diagram was used to design the backend which uses PostgreSQL; the system was designed using the Unified Modelling Language (UML). The business logic was implemented using Java platform Enterprise Edition. The application server used was WildFly 8.0. It was tested using some sample data to evaluate the reliability of the functional components of the system. The system takes into cognizance the temporal nature of data collected by nurses during the delivery of care to patients at point of care which make it possible to keep past, present and future records. A knowledge base for nursing was created using a template which will aid the proper implementation of evidence-based nursing, nursing research, nursing education, etc. The platform also automates the development and implementation of nursing care plan; and the management of the data collected in the course of administering care to patients. Furthermore, this system has fulfilled the requirement for distributing data collected during nursing process to all nurses who are authorized to have access to these records. This also enables nurses to work in a consistent environment without moving from one office to another in order collect records from other nurses. Therefore, this research has provided a new approach that will improve the quality of nursing care and proper documentation which will enhance nursing productivity; and provide a database for nursing research and quality assurance in nursing if implemented. It has also addressed issue of managing timevarying/temporal data in object relational data model.

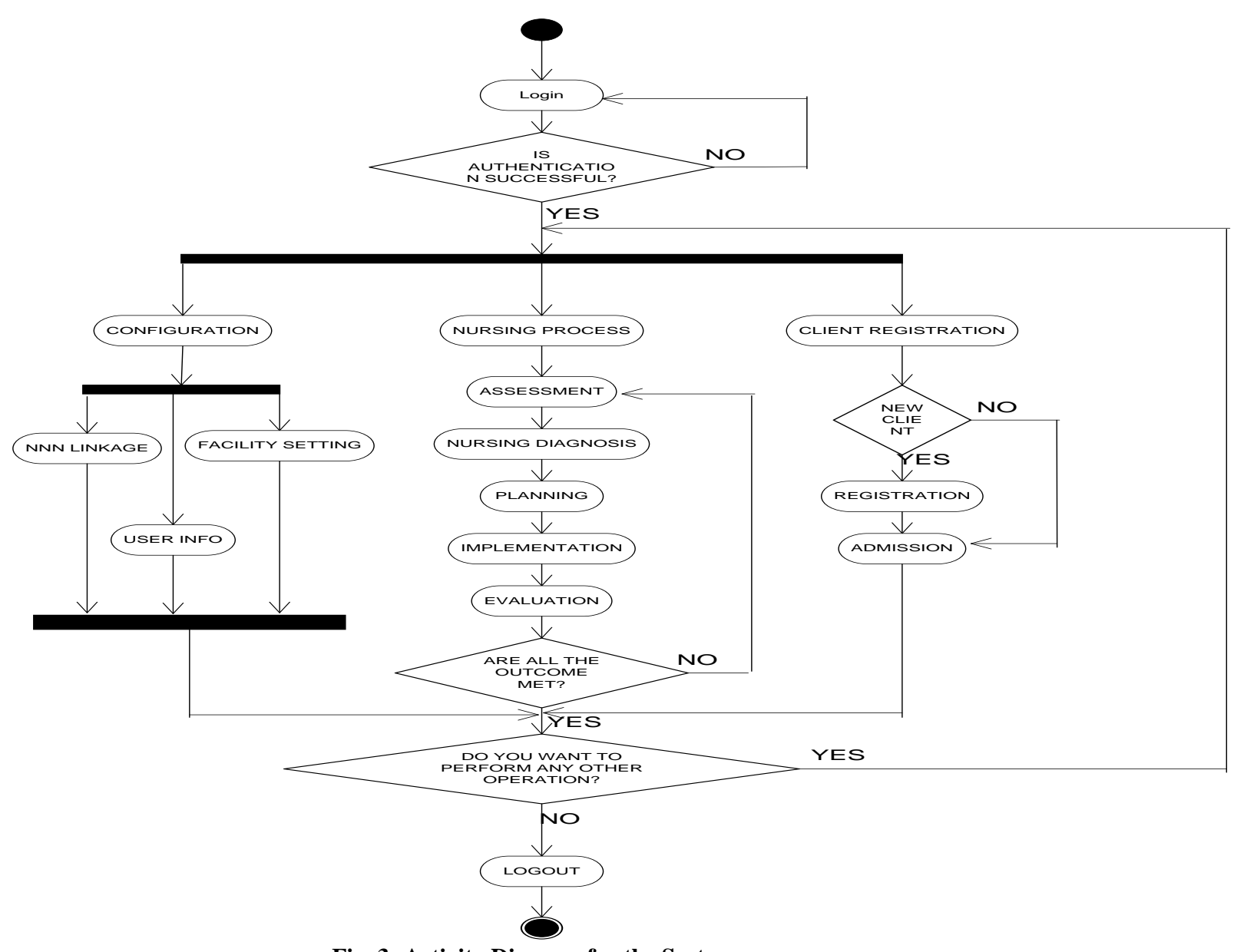

Fig. 3: Activity Diagram for the System 


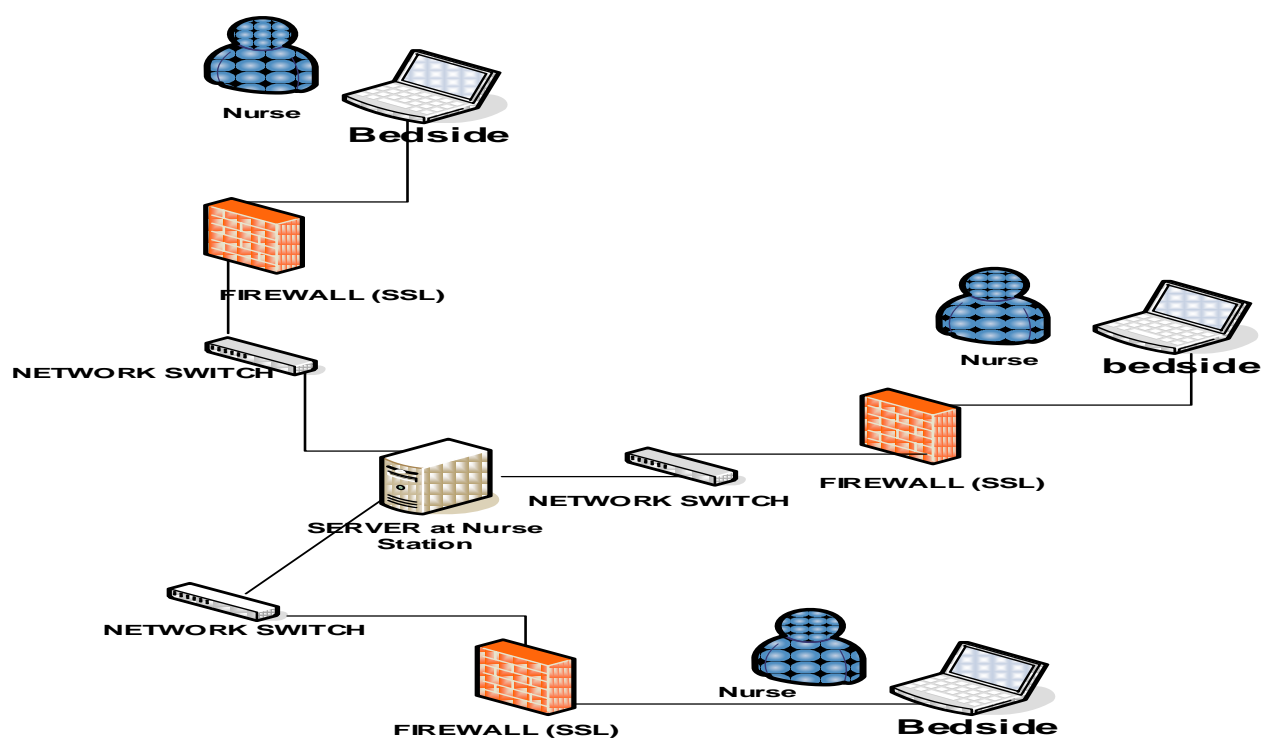

Fig. 4: Deployment view of the System

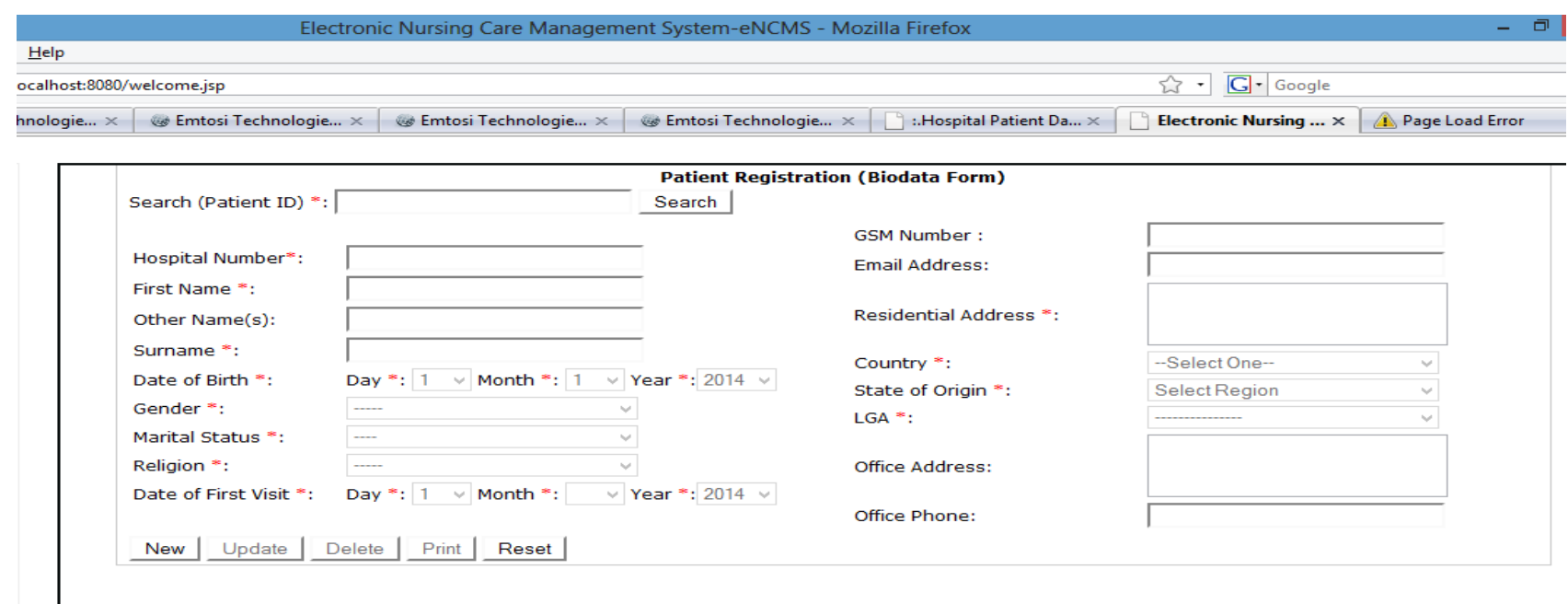

Fig. 5: Patient Biodata

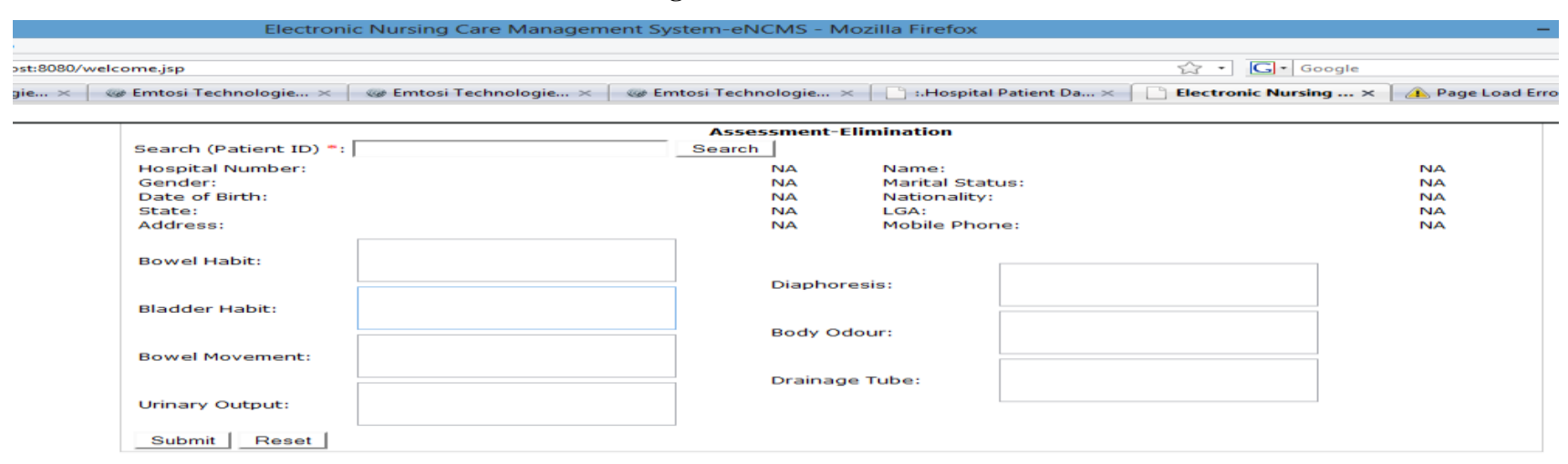

Fig. 6: Elimination 
Help

calhost:8080/welcome.jsp

¿. G. Google

Electronic Nursing Care Managem... $\times$

\begin{tabular}{|c|c|c|c|c|}
\hline \multicolumn{5}{|r|}{ Nursing Care Plan } \\
\hline Name: AVER & tina $\mathrm{A}$ & \multicolumn{2}{|c|}{ Date of Birth: 8-6-1980 } & Date of Admission: $28-05-2014$ 13:26:32 \\
\hline \multicolumn{5}{|c|}{ Diagnosis on Admission: $\mathrm{mp}$} \\
\hline \multicolumn{5}{|c|}{ Nursing Diagnosis: Impaired Gas Exchange, Feeding Self-Care Deficit } \\
\hline Date & Problem & Goal & Target Date & Nursing Orders \\
\hline $\begin{array}{l}09-07-2014 \\
00: 07: 22\end{array}$ & \begin{tabular}{|l} 
\#1 Impaired Gas \\
Exchange
\end{tabular} & $\begin{array}{l}\text { Ostomy Self-Care On } \\
\text { Likert Scale: } 1\end{array}$ & $\begin{array}{l}\text { 9-7-2014 } \\
\text { 9:30am }\end{array}$ & $\begin{array}{l}\text { Maintenance of an optimum urinary elimination pattern } \\
\text { TARFA Aper Simeon }\end{array}$ \\
\hline $\begin{array}{l}09-07-2014 \\
00: 18: 58\end{array}$ & $\begin{array}{l}\text { \#2 Feeding } \\
\text { Self-Care Deficit }\end{array}$ & $\begin{array}{l}\text { Activity Tolerance On } \\
\text { Likert Scale: } 3\end{array}$ & $\begin{array}{l}9-7-2014 \\
10: 15 a m\end{array}$ & $\begin{array}{l}\text { Prescription of and assistance with specific physical, cognitive, social, and spiritual activit } \\
\text { increase the range, frequency, or duration of an individual's (or group's) activity } \\
\text { ASSOH Kumawuese Adoo }\end{array}$ \\
\hline
\end{tabular}

Fig 7: Nursing Care Plan

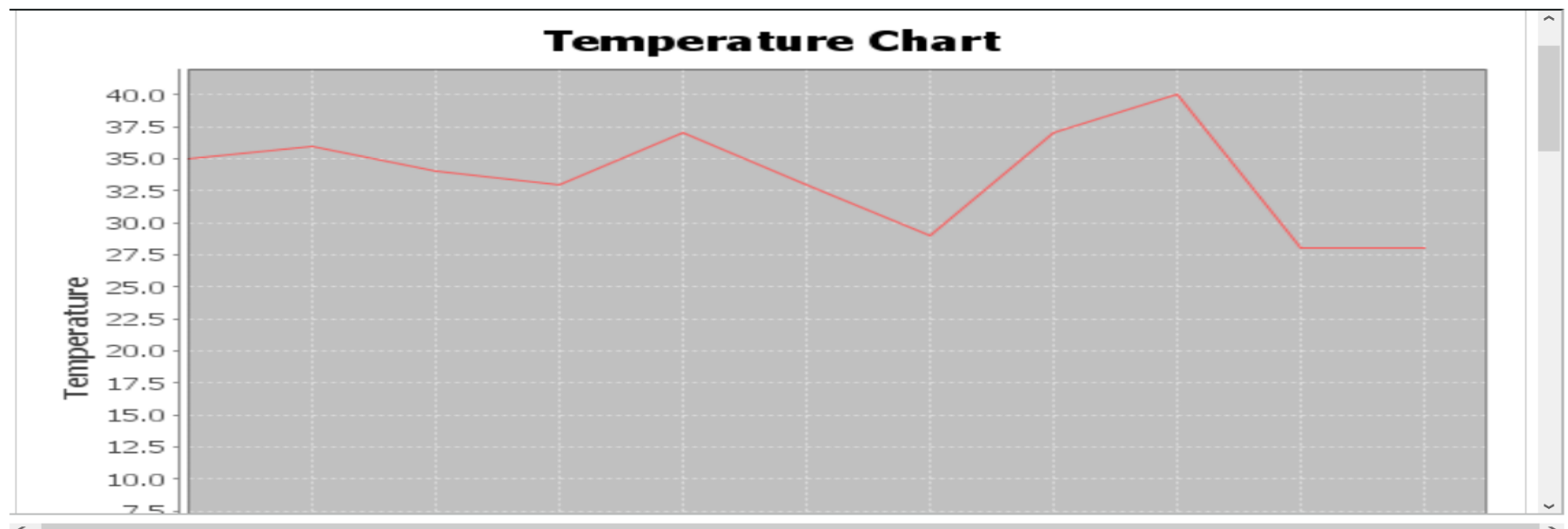

Fig 8: Temperature Chart

\section{Pulse Chart}

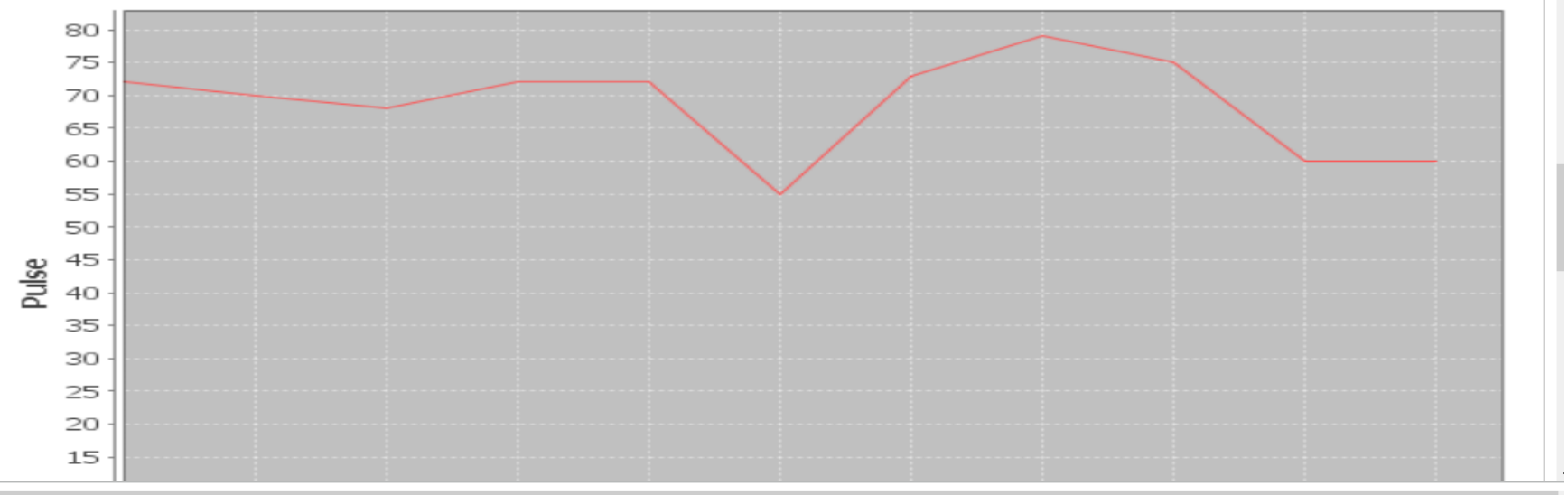

Fig 9: Pulse Chart 


\section{Respiration Chart}

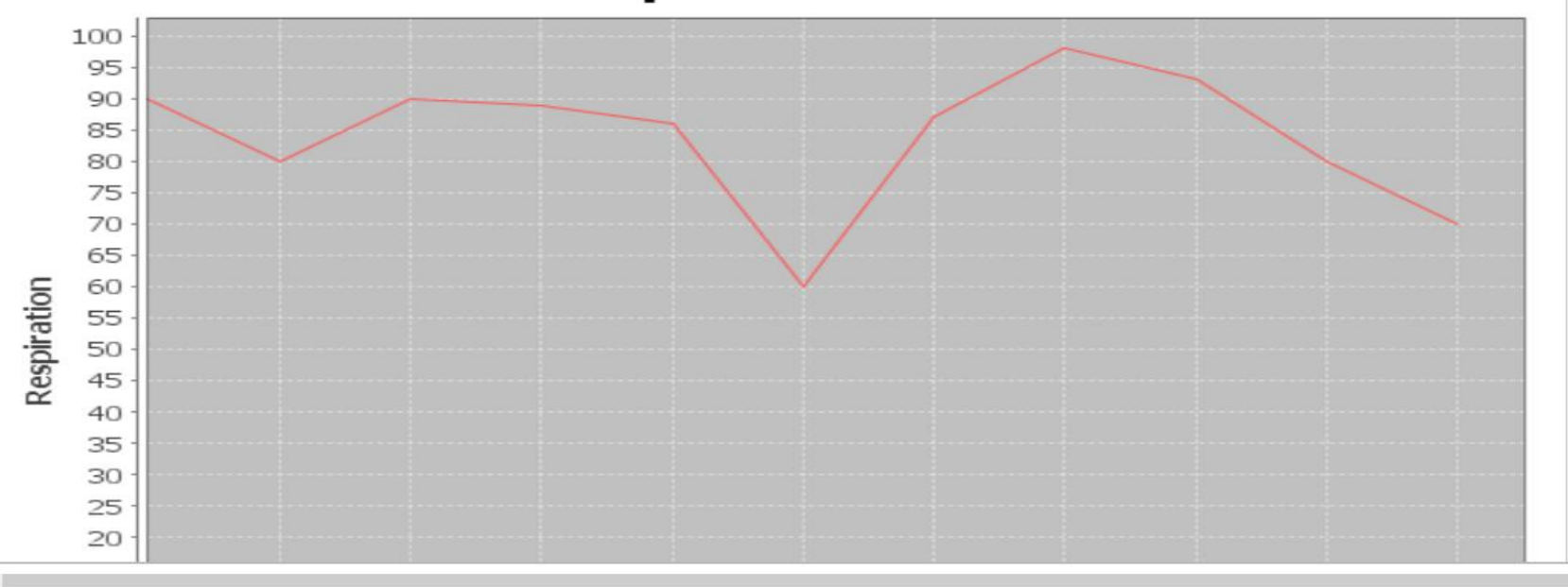

Fig 10: Respiration Chart

\section{REFERENCES}

[1] Lin, H. C., Hsu, M. H. and Yang, C. W. 2014. The Influences of Computer System Success and Informatics Competencies on Organizational Impact in Nursing Environments. Computers Informatics Nursing, 32(2): 90-99.

[2] Ayatollahi, H., P.A. Bath, PQ. A. and Goodacre, S. 2010. Factors influencing the use of IT in the Emergency Department: A Qualitative Study. Health Inform. J. 16 (3):189-200.

[3] Ackley, B. J. and Ladwig, G. B. 2008. Nursing diagnosis handbook: An evidence-based guide to planning care (8th ed.). St. Louis, MO: Mosby Elsevier.

[4] Ntsoele, M. 2011. An Evaluation of the Effective Use of Computer-Based Nursing Information System in Patient Care by Professional Nurses at Dr George Mukhari Hospital. MSc Dissertation. South Africa: Faculty of Health Sciences, University of Limpopo.

[5] Adeyemo F.O. and Olaogun, A.A. 2013. Factors affecting the use of Nursing Process in Health

Institutions in Ogbomoso Town, Oyo State. International Journal of Medicine and Pharmaceutical Sciences, 89-96.

[6] Afoi, B.B., Emmanuel, A., Garba, S.N., Gimba, S.M. and Afuwai, V. 2012. Evaluation of the Implementation of Nursing Process among Nurse Clinicians, Continental J. Nursing Science, 4(1), 34-46.

[7] Dong-Her, S., Hsiu-Sen, C. and Binshan, L. 2007. A Generalized Associative Petri Net for Reasoning, IEEE Transactions on Knowledge and Data Engineering, 19(9), 1241-1251.

[8] DiCenso, A., Guyatt, G. and Ciliska, D. 2005. Evidencebased nursing: A guide to clinical practice. Philadelphia: Elsevier.

[9] Burney, A., Ahsan, K., Mahmood, N. 2010. TempRPDM: A Conceptual Temporal Relational Model for Managing Patient Data. Recent Advances in Artificial Intelligence, Knowledge Engineering and Databases, 237-243.

[10] Combi, C., Juarez, J. M., Gozzi, M., Oliboni, B. and
Pozzi, G. (2007). Conceptual modelling of temporal clinical workflows. Proceedings of the International Workshop on Temporal Representation and Reasoning, 70-81.

[11] Price, A. M. 2013. Caring and Technology in an Intensive Care Unit: Anethnographic Study. Nurs Crit Care, 18(6): 278-88.

[12] Tunlind, A., Granstrom, J. and Engstrom, A. 2015. Nursing Care in a high Technological Environment: Experiences of Critical Care Nurses. Intensive Crit Care Nurs, 31(2):116-23.

[13] Momoh, M. A. and Chukwu, D. O. 2010. Factors that Militate Against the use of Nursing Process: A Hospital Based Study. Continental J. Pharmaceutical Sciences, 69.

[14] Bozak, M. 2003. Using Lewin's Force Field Analysis in Implementing a Nursing Information System. Computers, Informatics, Nursing, 80-85.

[15] Darbyshire, P. 2004. 'Rage against the Machine?': Nurses' and Midwives' Experiences of using Computerized Patient Information Systems for Clinical Information. Journal of Clinical Nursing, 17-25.

[16] Corkin, D. and Cardwell, P. 2012. The Nature of Care Planning and Nursing Delivery for Infants, Children and Young People. Lisa Hughes.

[17] Ogbuokiri, G.U. 2011. Challenges facing nursing in Nigeria award winning essay. School of Nursing, Ahmadu Bello University Teaching Hospital, Zaria, Kaduna State.

[18] Yange, S.T., Soriyan, H.A. and Olaogun, A.A. 2014 Conceptual view of a data model for nursing process. Proceedings of the 9th Health Informatics in Africa Conference (HELINA 2014), 17-25.

[19] Alexander, G. L. 2007. The nurse-patient trajectory framework. Studies in health technology and informatics, 129(2): 910-914. 